\title{
Incretin-based therapies and pancreatitis risk: myth or reality
}

\author{
Joseph M. Pappachan
}

Received: 17 November 2014/Accepted: 21 November 2014/Published online: 30 November 2014

(C) Springer Science+Business Media New York 2014

Incretins are gastrointestinal hormones secreted in response to food intake that modify biological mechanisms of glucose homeostasis in the body. Approximately $70 \%$ of insulin secretion by pancreatic $\beta$-cells is under the control of two incretin molecules, namely glucose-dependent intestinal polypeptide (GIP) and glucagon-like peptide-1 (GLP-1) [1]. Endogenous GLP-1 is rapidly degraded by the enzyme dipeptidyl peptidase-4 (DPP-4) to an inactive molecule that terminates its incretin effect. Research on the pharmacological manipulation of GLP-1 and DPP-4 molecules resulted in the development of multiple drugs in recent years, which are very useful for the treatment of type 2 diabetes mellitus (T2DM). These drugs, the GLP-1 analogues and DPP-4 inhibitors, form the incretin-based therapies in the management of T2DM, collectively termed as incretin mimetics or incretin analogues. With a modest effect on haemoglobin A1c, favourable effects on body weight and relatively low risk of hypoglycaemia [2], these drugs gained much popularity in the past few years. Incretin-based therapies are recommended by the American Diabetes Association (ADA) and the European Association for the Study of Diabetes (EASD) as second-line agents for the management of T2DM [2,3].

The meal-related insulin release from pancreas results from the binding of GLP-1 to $\beta$-cell GLP-1 receptors (GLP-1R). Because of the very short biological half-life, endogenously produced GLP-1 does not possess sustained effects on the pancreas. However, incretin mimetic drugs result in continued stimulation of GLP-1R with a potential for pleotropic effects on the pancreatic tissues [4].

J. M. Pappachan ( $₫)$

Department of Endocrinology, Walsall Manor Hospital, Walsall,

West Midlands WS2 9PS, UK

e-mail: drpappachan@yahoo.co.in
Experimental data from animal models showed conflicting results with some studies showing evidence of pancreatic damage from incretin mimetics $[5,6]$, whereas the others demonstrating protective effects $[7,8]$. Concerns were raised about the risk for pancreatic damage from incretinbased therapies based on multiple case reports and recent data from the United States Food and Drug Administration (FDA) adverse events reporting system. These prompted the FDA and other international agencies to caution the global medical fraternity about the potential harm related to the use of this new class of medications which were deemed to be promising in the management of T2DM, especially in obese individuals.

The warnings from different global agencies evoked a hot international debate on the safety of incretin-based therapies in 2013 [4, 9]. Several reports from the FDA and other international agencies also added chaos to the discussion although none has resolved the uncertainty completely. A systematic review and meta-analysis addressing this issue published early this year concluded that evidence for increased incidence of pancreatitis in T2DM patients taking incretin mimetics is inadequate, and that incretinbased therapies do not increase pancreatitis risk [10]. The studies analysed in this paper provided robust data from a total patient population of 33,350 cases treated for a median duration of 26 weeks that helped clearing the uncertainty to some extent.

Giorda et al. in this issue of Endocrine provide further evidence using a different search strategy on the lack of definite association between incretin-based therapies and pancreatitis risk through another systematic review and meta-analysis [11]. They analysed 136 abstracts from PubMed electronic database of which 15 full-text articles were reviewed in detail. A total of six papers from the systematic review were included in the meta-analysis. Four 
of these papers were cohort studies with a total of 82,569 patients exposed to exenatide, a GLP-1 analogue, and a total of 32,102 patients exposed to sitagliptin, a DPP-4 inhibitor. Two of the articles were case-controlled studies with a total number of 1,177 patients treated with exenatide or sitagliptin. Pooled odds ratio (OR) of 0.94 [95\% confidence interval (95\% CI): 0.76-1.17] was obtained from the cohort studies and 1.41 (95\% CI: 0.68-2.94) from the case-controlled studies, with an overall OR of 1.08 (95\% CI: 0.84-1.40) for pancreatitis in patients receiving incretin-based therapies.

Results of this meta-analysis also provide the global scientific community reasonable reassurance on the safety of incretin-based therapies. These studies [10, 11] shall clearly weaken the impact of propaganda about the very high risk of pancreatitis of more than ten-fold from incretin mimetic drugs projected through adverse event reporting systems [12]. Alerts from the FDA and European agencies about high risk of pancreatitis followed by widespread media coverage on the matter created a big pandemonium globally among the public on the safety of these medications. We are now equipped with better data to explain the logic of using incretin-based drugs which have clearly proven benefits in obese patients with T2DM.

However, we should also be vigilant about the potential long-term effects of this new class of drugs. At the moment, we are unaware of the implications of chronic GLP-1R stimulation and its effects on enzyme synthesis and the potential to evoke inflammatory response in the human pancreas. Marked $\beta$-cell hyperplasia, co-expression of insulin and glucagon from $\beta$-cells, $\alpha$-cell hyperplasia, excess proliferation markers and higher prevalence of preneoplastic lesions were observed in the pancreas specimens of organ donors previously treated with incretin mimetics for T2DM [13]. Although these data are from a small study, it should be an eye opener to the scientific community to be cautious about the potential for long-term pleotropic effects of these medications on the pancreas.

Animal models clearly demonstrated the pleotropic effects of incretin mimetics on the pancreas, which lead on to proliferative changes in acinar and ductal cells, metaplasia, potentially pre-malignant changes and increased pancreatic weight [4]. Considering the short life spans of laboratory animals and the species-specific variations in drug effects, the long-term implications of above observations are still unclear. More than the scare about hypothetical risk of acute and chronic pancreatitis, the probability of pancreatic neoplasms should be a serious consideration in this context. Subclinical and full-blown pancreatitis are well-known risk factors for pancreatic cancer [4], and there are some signals about this possibility from different studies [4, 14].
We should also consider other potential risk factors for pancreatitis and pancreatic cancer in patients taking incretin-based therapies. The majority of patients treated with these drugs are overweight or obese. It is well known that obesity is a major risk factor for pancreatitis and pancreatic cancer [15]. Obesity induces a chronic inflammatory state through complex mechanisms that lead on to increased risk and severity of pancreatitis. Epidemiologic and cohort studies showed that obesity is a significant risk factor for pancreatic carcinogenesis through some obscure mechanisms $[15,16]$. This association is another grey area that needs further research before we attribute possible causal links between pancreatitis, pancreatic cancer and incretin-based therapies.

It should be borne in mind that T2DM itself is a risk factor for pancreatic cancer. A large cohort study comparing 1,10,919 cases and 2,11,695 controls showed a strong etiological association of T2DM and hyperinsulinemia in the pathogenesis of pancreatic cancer [17]. A meta-analysis of three large cohort studies demonstrated a 1.8-fold increased risk of pancreatic cancer in patients with T2DM [18]. Therefore, we should be cautious regarding the use of these medications which may have a propensity to increase the risk of pancreatic cancer.

Another factor to be considered is the effects of incretin analogues on GLP-1R in organs other than the pancreas which are not yet well researched. GLP-1R is widely distributed in the brain (brainstem, hypothalamus, hippocampus and cortical nuclei) and peripheral tissues (enteric nerves, vagal nerves, stomach, small and large bowel and adipose tissue) [19]. Because of rapid degradation by the DPP-4 enzyme, the endogenously produced GLP-1 may have only focal and short lived hormonal effects in the gut and pancreas, whereas the GLP-1 manipulation by incretin analogues may cause sustained stimulation of GLP-1R in a wide variety of body tissues. The implications of this effect of incretin-based therapy is another area of uncertainty that needs further clarification especially in the background of alert about possible association between thyroid cancer and incretin analogues $[14,20]$.

Yet another factor to be considered is the potential harm which can be associated with advanced organ failure such as renal and hepatic failure that can complicate diabetes. Although incretin mimetics are claimed to be safe in mild renal impairment, the safety in moderate to severe kidney disease and hepatic disease needs further verification as suggested by a recent systematic review [21].

On the contrary, Gallwitz recently suggested extrapancreatic beneficial effects of incretin manipulation in a detailed review [22]. Cardio-protective effects of GLP-1 agonist therapy have been demonstrated in ischemia models in preclinical data and short-term small studies. Lowering of blood pressure is a well-known positive effect 
of therapy with GLP-1 analogues. Studies in animals showed some beneficial effects of incretin mimetics in neurological diseases such as Parkinson's disease, Alzheimer's disease and stroke although these findings need verification with large-scale clinical trial data for making logical practice recommendations.

We were confronted with a dilemma of whether to continue or to discard a promising class of medications significantly useful in the management of obese patients with T2DM when different international agencies such as the FDA alerted the global medical fraternity about the potential harmful effects related to incretin analogues. The study reported by Giorda et al. in this issue of Endocrine helps us to be more reassured about the safety of incretinbased therapies in the management of T2DM [11]. However, we should also keep in mind that our experience of slightly more than a decade is still insufficient to judge the long-term safety of this class of medications that may have effects in many body tissues. Therefore, constant vigilance from the global scientific community regarding this matter is essential to ensure that we adopt the right track.

Conflict of interest There are no conflicts in interest related to this article.

\section{References}

1. L.L. Baggio, D.J. Drucker, Biology of incretins: GLP-1 and GIP. Gastroenterology 132, 2131-2157 (2007)

2. S.E. Inzucchi, R.M. Bergenstal, J.B. Buse, M. Diamant, E. Ferrannini, M. Nauck et al., Management of hyperglycaemia in type 2 diabetes: a patient-centered approach. Position statement of the American Diabetes Association (ADA) and the European Association for the Study of Diabetes (EASD). Diabetologia 55, 1577-1596 (2012)

3. American Diabetes Association, Standards of medical care in diabetes-2014. Diabetes Care 37, S14-S80 (2014)

4. P.C. Butler, M. Elashoff, R. Elashoff, E.A. Gale, A critical analysis of the clinical use of incretin-based therapies: are the GLP-1 therapies safe? Diabetes Care 36, 2118-2125 (2013)

5. J.S. Nachnani, D.G. Bulchandani, A. Nookala, B. Herndon, A. Molteni, P. Pandya et al., Biochemical and histological effects of exendin-4 (exenatide) on the rat pancreas. Diabetologia 53, 153-159 (2010)

6. B. Gier, A.V. Matveyenko, D. Kirakossian, D. Dawson, S.M. Dry, P.C. Butler, Chronic GLP-1 receptor activation by exendin-4 induces expansion of pancreatic duct glands in rats and accelerates formation of dysplastic lesions and chronic pancreatitis in the Kras(G12D) mouse model. Diabetes 61, 1250-1262 (2012)

7. K. Tatarkiewicz, P.A. Smith, E.J. Sablan, C.J. Polizzi, D.E. Aumann, C. Villescaz et al., Exenatide does not evoke pancreatitis and attenuates chemically induced pancreatitis in normal and diabetic rodents. Am. J. Physiol. Endocrinol. Metab. 299, E1076E1086 (2010)

8. J.A. Koehler, L.L. Baggio, B.J. Lamont, S. Ali, D.J. Drucker, Glucagon-like peptide-1 receptor activation modulates pancreatitis-associated gene expression but does not modify the susceptibility to experimental pancreatitis in mice. Diabetes 58, 2148-2161 (2009)

9. M.A. Nauck, A critical analysis of the clinical use of incretinbased therapies: the benefits by far outweigh the potential risks. Diabetes Care 36, 2126-2132 (2013)

10. L. Li, J. Shen, M.M. Bala, J.W. Busse, S. Ebrahim, P.O. Vandvik et al., Incretin treatment and risk of pancreatitis in patients with type 2 diabetes mellitus: systematicreview and meta-analysis of randomised and non-randomised studies. BMJ 348, 2366 (2014)

11. C.B. Giorda, C. Sacerdote, E. Nada, L. Marafetti, I. Baldi, R. Gnavi, Incretin-based therapies and acute pancreatitis risk: a systematic review and meta-analysis of observational studies. Endocrine (2014). doi:10.1007/s12020-014-0386-8

12. J.L. Faillie, S. Babai, S. Crépin, V. Bres, M.L. Laroche, H. Le Louet et al., Pancreatitis associated with the use of GLP-1 analogs and DPP-4 inhibitors: a case/non-case study from the French Pharmacovigilance Database. Acta Diabetol. 51, 491-497 (2014)

13. A.E. Butler, M. Campbell-Thompson, T. Gurlo, D.W. Dawson, M. Atkinson, P.C. Butler, Marked expansion of exocrine and endocrine pancreas with incretin therapy in humans with increased exocrine pancreas dysplasia and the potential for glucagon-producing neuroendocrine tumors. Diabetes 62, 2595-2604 (2013)

14. M. Elashoff, A.V. Matveyenko, B. Gier, R. Elashoff, P.C. Butler, Pancreatitis, pancreatic, and thyroid cancer with glucagon-like peptide-1-based therapies. Gastroenterology 141, 150-156 (2011)

15. I. Gukovsky, N. Li, J. Todoric, A. Gukovskaya, M. Karin, Inflammation, autophagy, and obesity: common features in the pathogenesis of pancreatitis and pancreatic cancer. Gastroenterology 144, 1199-1209 (2013)

16. M. Wiseman, The second World Cancer Research Fund/American Institute for Cancer Research expert report. Food, nutrition, physical activity, and the prevention of cancer: a global perspective. Proc. Nutr. Soc. 67, 253-256 (2008)

17. A. Yacoub, E. Siegel, E. Makhoul, Pancreatic cancer and diabetes mellitus: a retrospective cohort study. J. Clin. Oncol. 29(Suppl), $4102(2011)$

18. D. Li, H. Tang, M.M. Hassan, E.A. Holly, P.M. Bracci, D.T. Silverman, Diabetes and risk of pancreatic cancer: a pooled analysis of three large case-control studies. Cancer Causes Control 22, 189-197 (2011)

19. M.J. Dailey, T.H. Moran, Glucagon-like peptide 1 and appetite. Trends Endocrinol. Metab. 24, 85-91 (2011)

20. Y. Cao, XM. Liu, Should we still be concerned about the potential side effects of glucagon-like peptide-1 receptor agonists on thyroid C cells? Endocrine (2014). doi:10.1007/s12020-0140354-3

21. C.B. Giorda, E. Nada, B. Tartaglino, Pharmacokinetics, safety, and efficacy of DPP-4 inhibitors and GLP-1 receptor agonists in patients with type 2 diabetes mellitus and renal or hepatic impairment. A systematic review of the literature. Endocrine 46, 406-419 (2014)

22. B. Gallwitz, Extra-pancreatic effects of incretin-based therapies. Endocrine 47, 360-371 (2014) 